\title{
Continuation of Double Hopf Points in Thermal Convection of Rotating Fluid Spheres*
}

\author{
J. Sánchez Umbría ${ }^{\dagger}$ and $M$. Net $^{\dagger}$
}

\begin{abstract}
The thermal convection of rotating fluids in spherical geometry is a classical problem with application to many geophysical and astrophysical problems. The study of the transition to periodic solutions from the steady conduction state of a rotating and self-gravitating fluid sphere, heated uniformly from the inside, is discussed here. The continuation of double Hopf points is used to determine the region of the parameter space in which the first bifurcation is to solutions independent of the longitude (axisymmetric solutions). It is limited by three segments of curves separated by two triple Hopf points. This type of so-called torsional solutions was recently found, and it is shown here that they are the preferred solutions at the onset of convection for a wide range of fluids of Prandtl numbers, $\operatorname{Pr}$, extending from $\operatorname{Pr}=0$ to $\operatorname{Pr} \approx 0.9$, which includes, for instance, liquid metals and gases. Although the corresponding interval of Ekman numbers, E, narrows when $\operatorname{Pr} \rightarrow 0$, it is shown that there is always a small gap of parameters, relevant to geophysics and astrophysics, where the torsional solutions are preferred. The limits of the double Hopf curves when $\operatorname{Pr} \rightarrow 0$ follow linear laws of the form $\mathrm{E}=c\left(m_{1}, m_{2}\right) \operatorname{Pr}, c\left(m_{1}, m_{2}\right)$ being constant depending on the two azimuthal wavenumbers, $m_{1}$ and $m_{2}$, of the eigenfunctions that define the double Hopf problem.
\end{abstract}

Key words. continuation methods, double Hopf bifurcation, thermal convection, rotating fluids, stability analysis

AMS subject classifications. 34C23, 34C25, 76D05, 76E06, 76E20

DOI. $10.1137 / 20 \mathrm{M} 1333961$

1. Introduction. The combined action of rotation, and thermal and solutal convection plays a fundamental role in the dynamics of the interiors and atmospheres of most planets and stars, and in the generation of self-sustaining magnetic fields. Such effects are responsible for a great variety of phenomena in a wide range of scales, for instance, for the mean zonal flows observed in the atmospheres of the giant planets or the small-scale granulation observed in the upper convective layer of the Sun. The differential rotation driven by the convection is also responsible for the dipolar magnetic field measured in the Earth's surface or for the smallscale concentrations of the magnetic field, appearing as dark sunspots, due to the inhibition of the convection in the photosphere of the Sun.

The study of the physics of these phenomena is complicated because of the scarcity of observations and experimental measurements, and also because of the complexity and cost of the numerical simulations. Moreover, each celestial body has its own peculiarities. Nowadays

\footnotetext{
* Received by the editors April 27, 2020; accepted for publication (in revised form) by H. Osinga November 10, 2020; published electronically DATE.

https://doi.org/10.1137/20M1333961

Funding: This work was supported by Spanish MINECO/FEDER under grant FIS2016-76525-P and by Catalan AGAUR under grant 2017-SGR-1374.

${ }^{\dagger}$ Departament de Física, Universitat Politècnica de Catalunya, 08034 Barcelona, Spain (juan.j.sanchez@upc.edu, marta.net@upc.edu).
} 
it is impossible to reach the parameters that quantify their properties, either in the numerical simulations or in laboratory experiments, so there are a lot of basic open problems on geophysical and astrophysical topics and on magnetohydrodynamics. They are usually approached in several ways. Aside from the theoretical studies, the data achieved from the numerical simulations, although using parameters far from the real values, together with those of the direct observations supply the information needed to build reduced models such as amplitude equations [22] or to apply data assimilation methods for the forecast of different events [36].

The computational cost of the direct numerical simulations is always very high due to multiple factors that converge in each phenomenon, and to the extreme values of the parameters. The simplest system of partial differential equations (PDEs), governing the thermal convection in rotating fluids, is given by the Boussinesq approximation of the mass, momentum, and energy equations, which consists in considering the dependence of the density with the temperature only in the buoyancy term, and in keeping the rest of the physical constants independent of the point. Even in this case, the nondimensional equations for a full sphere depend on three parameters: E, Pr, and the Rayleigh, Ra, numbers. The first is $10^{-16}<\mathrm{E}<10^{-7}$ in the celestial bodies, and the second is estimated to be $\operatorname{Pr}<1$. In addition, in the case of spherical shells the radius ratio (inner over outer radius) of the shell gives rise to a fourth parameter. In magnetohydrodynamics the induction equation must be added to this system, introducing a new parameter, the magnetic Reynolds number, $\mathrm{R}_{\mathrm{m}}$, or the magnetic Prandtl number, $\mathrm{Pr}_{\mathrm{m}}$, depending of the nondimensional scales used. They also have unreachable values, $\mathrm{R}_{\mathrm{m}}>10^{3}$ and $\operatorname{Pr}_{\mathrm{m}}<10^{-5}$, in the simulations.

The extrapolation of the data supplied by the direct numerical simulations, computed at the ranges of achievable parameters, gives valuable information on the mean values of physical magnitudes such as energies, zonal flows, vorticities, time scales, etc. [9, 2, 24, 30]. The patterns of convection and magnetic fields are parameter dependent, and, at high Ra, they often retain a remnant of the primary patterns from which they bifurcate, even in the chaotic and turbulent states [19], so it is important to determine the range of parameters where each pattern may appear, and where it is stable or not. The linear stability analysis of the solutions of the equations of fluid mechanics is the most efficient way to do it and, in addition, provides insight into the physical mechanisms leading to increasingly more complex flows. It is one of the main tools in the field of hydrodynamic stability, which in most cases are those of the dynamical systems. Invariant objects such as equilibria, periodic orbits, tori, and homoand heteroclinic orbits are found whenever it is possible, and their stability is studied. There is a large volume of literature on the subject. We refer the reader to $[8,32,33,18,54,12]$ just to mention a few remarkable books from the sixties to the present.

We focus in this article on the thermal convection of a pure fluid sphere rotating about a fixed axis and with uniform internal heating. It is a simplified model of many celestial bodies or part of their interiors, but which is rich enough to reproduce behaviors and patterns observed in real situations. Numerical simulations and experimental studies trying to mimic full spherical bodies are not as abundant as those in the case of spherical shells (see [7, 6, 56, 55, 10, 26, 22], among many others). The topics analyzed go from the study of the stability of the trivial conduction state to the simulation of fully turbulent flows, in some cases coupled with the induction equation.

The conduction state, as will be seen later, depends only on the radial coordinate, and the 
study of its linear stability leads to a non-self-adjoint problem. The most common situation is to have a Hopf bifurcation breaking the rotational invariance, i.e., the eigenfunctions have longitudinal dependence but are still symmetric with respect to the equator. Under these conditions the bifurcation leads generically to azimuthal rotating waves [27]. This case has been studied extensively for spherical shells by using numerical methods or trying to formulate asymptotic theories in several distinguished limits (see $[44,4,7,58,59,31,5,17,1,23,41$, $3,42]$, among others). A new type of solutions, so-called torsional, was found numerically by solving the stability problem for stress-free boundary conditions [49]. It was shown that at low $\operatorname{Pr}$ and for ratios $\operatorname{Pr} / \mathrm{E}=\mathcal{O}(10)$ the eigenfunctions at the first bifurcation are azimuthally axisymmetric and antisymmetric with respect to the equator. This result was confirmed soon by means of asymptotic methods [60]. The torsional linear solutions give rise to an oscillation between both hemispheres. Following these linear stability analyses, the bifurcated branches of solutions have also been computed [37, 53]. It was shown that the torsional flows also produce a latitudinal kinetic energy wave propagating near and on the surface of the sphere.

In order to find the curves delimiting the region of first transitions to torsional solutions, the continuation of Hopf and double Hopf points is used here. Continuation methods have been used in fluid mechanics soon after the appearance of seminal works on the subject [38, 35]. Early examples of application, for instance, to the classical Taylor-Couette problem, using in some cases initial versions of the well-known package AUTO [15], can be found in $[40,11,14]$. In particular, the work of Cliffe [11] included the continuation of saddle-node and pitchfork bifurcation points, and even the detection of cusp points. Several review articles and books describe the status and the history of the use of dynamical system techniques in fluid mechanics, and how these methods can help to understand the transition to turbulence (see $[16,34,13,51,25]$ and the introduction of [43]). The continuation of codimension-one bifurcations of equilibria for PDEs has been included in numerical packages such as LOCA [48], and pde2path [57].

The goal of the present work is twofold: to lay out an algorithm for the computation of double Hopf points in large-scale dissipative systems, and to determine the region of the parameter space in which the first bifurcation from the conduction state is to solutions independent of the longitudinal coordinate, in order to find out its possible relevance in the dynamics observed in planets and stars.

The layout of the paper is as follows: Section 2 describes the formulation of the thermal convection problem, and the numerical techniques used to discretize it. In section 3 the extended systems used to track the curves of Hopf and double Hopf points are presented. Section 5 shows the bifurcation curves found, as well as the final region in parameter space in which the first bifurcation from the conduction state is to axisymmetric torsional solutions. Some final remarks and conclusions are stated in section 6. Appendix A contains details on the numerical continuation techniques employed to track the curves of Hopf and double Hopf points.

2. Mathematical model and numerical discretization. Let us consider the thermal convection of a fluid sphere of radius $r_{o}$, internally and uniformly heated, rotating about an axis of symmetry of direction $\mathbf{k}$ (unitary) with constant angular velocity $\boldsymbol{\Omega}=\Omega \mathbf{k}$, and subject to radial gravity $\mathbf{g}=-\gamma \mathbf{r}$, where $\gamma$ is a constant and $\mathbf{r}$ the position vector. This is the 
gravity field inside a spherical mass of uniform density. The momentum, mass, and energy equations are written, using the Boussinesq approximation, in a frame of reference rotating with angular velocity $\Omega$. Spherical coordinates, $(r, \theta, \varphi)$, with $\theta$ measuring the colatitude and $\varphi$ the longitude, are used. In addition, the density in the Coriolis term is taken constant, and the centrifugal force is neglected, since it is small compared to gravity in many celestial bodies such as, for instance, in the major planets, i.e., $\Omega^{2} / \gamma \ll 1$. The scales used to write the dimensionless equations are $r_{o}$ for the distance, $\nu^{2} / \gamma \alpha r_{o}^{4}$ for the temperature, and $r_{o}^{2} / \nu$ for the time, $\nu$ and $\alpha$ being the kinematic viscosity and the thermal expansion coefficient, respectively. Therefore the nondimensional equations are

$$
\begin{aligned}
\partial_{t} \mathbf{v}+(\mathbf{v} \cdot \nabla) \mathbf{v}+2 \mathrm{E}^{-1} \mathbf{k} \times \mathbf{v} & =-\nabla p+\nabla^{2} \mathbf{v}+\Theta \mathbf{r} \\
\nabla \cdot \mathbf{v} & =0 \\
\operatorname{Pr}\left(\partial_{t} \Theta+(\mathbf{v} \cdot \nabla) \Theta\right) & =\nabla^{2} \Theta+\operatorname{Ra} \mathbf{r} \cdot \mathbf{v}
\end{aligned}
$$

where $\mathbf{v}, p$, and $\Theta=T-T_{c}$ are the velocity field, the modified pressure, and the temperature perturbation from the conduction state, respectively. The latter is, in these nondimensional units, $\mathbf{v}_{c}=\mathbf{0}$ and $T_{c}(r)=T_{0}-(\mathrm{Ra} / 2 \mathrm{Pr}) r^{2}$, with $T_{0}$ an arbitrary temperature of reference. The nondimensional parameters of the problem are the Rayleigh (Ra), Ekman (E), and Prandtl (Pr) numbers, defined as

$$
\mathrm{Ra}=\frac{\beta \gamma \alpha r_{o}^{6}}{\kappa \nu}, \quad \mathrm{E}=\frac{\nu}{\Omega r_{o}^{2}}, \quad \operatorname{Pr}=\frac{\nu}{\kappa},
$$

where $\beta=q / 3 \kappa c_{p}, q$ is the (uniform) rate of heat generation per unit mass, $\kappa$ is the thermal diffusivity, and $c_{p}$ is the specific heat at constant pressure. In the above system $2 \mathrm{E}^{-1} \mathbf{k} \times \mathbf{v}$ is the Coriolis term; Ra $\mathbf{r} \cdot \mathbf{v}$ comes from writing the temperature equation around the basic conduction state, i.e., it appears when $T$ is substituted by $T=T_{c}+\Theta$ in its original equation $\left(\operatorname{Pr}\left(\partial_{t} T+(\mathbf{v} \cdot \nabla) T\right)=\nabla^{2} T\right)$ to obtain that for $\Theta$. Moreover $\Theta \mathbf{r}$ is the forcing term responsible for the thermal instability. It depends on $\Theta$ after taking into account the dependence of the density on $T$ in the gravity term, and writing $T=T_{c}+\Theta$.

The divergence-free velocity field is then expressed in terms of toroidal, $\Psi$, and poloidal, $\Phi$, potentials (see [8]) as

$$
\mathbf{v}=\nabla \times(\Psi \mathbf{r})+\nabla \times \nabla \times(\Phi \mathbf{r}) .
$$

The equations for both potentials are obtained by taking the radial component of the curl and double curl of the momentum equation. After rewriting also that of the temperature, the final system, linearized around the basic state $(\Psi, \Phi, \Theta)=(0,0,0)$, is

$$
\begin{aligned}
\left(\partial_{t}-\nabla^{2}\right) \mathcal{L}_{2} \Psi & =2 \mathrm{E}^{-1}\left(\partial_{\varphi} \Psi-\mathcal{Q} \Phi\right), \\
\left(\partial_{t}-\nabla^{2}\right) \mathcal{L}_{2} \nabla^{2} \Phi & =2 \mathrm{E}^{-1}\left(\partial_{\varphi} \nabla^{2} \Phi+\mathcal{Q} \Psi\right)-\mathcal{L}_{2} \Theta, \\
\left(\operatorname{Pr} \partial_{t}-\nabla^{2}\right) \Theta & =\operatorname{Ra} \mathcal{L}_{2} \Phi .
\end{aligned}
$$

The operators $\mathcal{L}_{2}$ and $\mathcal{Q}$ are defined by

$$
\mathcal{L}_{2}=-r^{2} \nabla^{2}+\partial_{r}\left(r^{2} \partial_{r}\right), \quad \mathcal{Q}=r \cos \theta \nabla^{2}-\left(\mathcal{L}_{2}+r \partial_{r}\right)\left(\cos \theta \partial_{r}-r^{-1} \sin \theta \partial_{\theta}\right) .
$$


Stress-free and perfect thermally conducting boundary conditions are assumed at $r=1$, which become

$$
\partial_{r}(\Psi / r)=0, \quad \Phi=0, \quad \partial_{r r}^{2} \Phi=0, \quad \text { and } \quad \Theta=0
$$

in terms of the velocity potentials and the temperature perturbation.

To discretize the equations the functions $X=(\Psi, \Phi, \Theta)$ are first expanded in spherical harmonic series with a triangular truncation of maximal degree $L$, namely,

$$
X(t, r, \theta, \varphi)=\sum_{l=0}^{L} \sum_{m=-l}^{l} X_{l}^{m}(r, t) Y_{l}^{m}(\theta, \varphi),
$$

with $\Psi_{l}^{-m}=\overline{\Psi_{l}^{m}}, \Phi_{l}^{-m}=\overline{\Phi_{l}^{m}}, \Theta_{l}^{-m}=\overline{\Theta_{l}^{m}}$, and imposing $\Psi_{0}^{0}=\Phi_{0}^{0}=0$ to uniquely determine the two scalar potentials. The spherical harmonics are normalized as

$$
Y_{l}^{m}(\theta, \varphi)=\sqrt{\frac{2 l+1}{2} \frac{(l-m) !}{(l+m) !}} P_{l}^{m}(\cos \theta) e^{i m \varphi}, \quad l \geq 0, \quad 0 \leq m \leq l,
$$

$P_{l}^{m}$ being the associated Legendre functions of degree $l$ and order $m$. Equations (2.3)-(2.5) written for the complex coefficients become

$$
\begin{aligned}
\partial_{t} \Psi_{l}^{m} & =\mathcal{D}_{l} \Psi_{l}^{m}+\frac{2 \mathrm{E}^{-1}}{l(l+1)}\left(i m \Psi_{l}^{m}-[\mathcal{Q} \Phi]_{l}^{m}\right), \\
\partial_{t} \mathcal{D}_{l} \Phi_{l}^{m} & =\mathcal{D}_{l}^{2} \Phi_{l}^{m}-\Theta_{l}^{m}+\frac{2 \mathrm{E}^{-1}}{l(l+1)}\left(i m \mathcal{D}_{l} \Phi_{l}^{m}+[\mathcal{Q} \Psi]_{l}^{m}\right), \\
\partial_{t} \Theta_{l}^{m} & =\operatorname{Pr}^{-1} \mathcal{D}_{l} \Theta_{l}^{m}+\operatorname{Pr}^{-1} l(l+1) \operatorname{Ra} \Phi_{l}^{m},
\end{aligned}
$$

with

$$
\mathcal{D}_{l}=\partial_{r r}^{2}+\frac{2}{r} \partial_{r}-\frac{l(l+1)}{r^{2}} .
$$

The boundary conditions decouple for each degree and order, i.e.,

$$
\partial_{r}\left(\Psi_{l}^{m} / r\right)=0, \quad \Phi_{l}^{m}=0, \quad \partial_{r r}^{2} \Phi_{l}^{m}=0, \quad \text { and } \quad \Theta_{l}^{m}=0
$$

at $r=1$. The square bracket $[\cdot]_{l}^{m}$ indicates extracting the spherical harmonic coefficient of degree $l$ and order $m$. The operator $\mathcal{Q}$ is

$$
[\mathcal{Q} f]_{l}^{m}=-(l-1)(l+1) c_{l}^{m} D_{1-l}^{+} f_{l-1}^{m}-l(l+2) c_{l+1}^{m} D_{l+2}^{+} f_{l+1}^{m},
$$

$$
\text { with } \quad D_{l}^{+}=\partial_{r}+\frac{l}{r}, \quad \text { and } \quad c_{l}^{m}=\left(\frac{l^{2}-m^{2}}{4 l^{2}-1}\right)^{1 / 2}
$$


To study the stability of $(\Psi, \Phi, \Theta)=0$ the eigenvalue problems

$$
\begin{aligned}
\lambda \Psi_{l}^{m} & =\mathcal{D}_{l} \Psi_{l}^{m}+\frac{1}{l(l+1)}\left[2 \mathrm{E}^{-1}\left(i m \Psi_{l}^{m}-[\mathcal{Q} \Phi]_{l}^{m}\right)\right], \\
\lambda \mathcal{D}_{l} \Phi_{l}^{m} & =\mathcal{D}_{l}^{2} \Phi_{l}^{m}-\Theta_{l}^{m}+\frac{1}{l(l+1)}\left[2 \mathrm{E}^{-1}\left(i m \mathcal{D}_{l} \Phi_{l}^{m}+[\mathcal{Q} \Psi]_{l}^{m}\right)\right], \\
\lambda \Theta_{l}^{m} & =\operatorname{Pr}^{-1} \mathcal{D}_{l} \Theta_{l}^{m}+\operatorname{Pr}^{-1} l(l+1) \operatorname{Ra} \Phi_{l}^{m}
\end{aligned}
$$

must be solved for $m=0,1, \ldots, \lambda$ being the eigenvalue. The eigenproblems for negative $m$ have conjugated eigenvalues and eigenvectors of those for positive $m$. The case $m=0$ is special since it is real and eigenvalues come in conjugated pairs.

Since the operator $\mathcal{Q}$ only couples the spherical harmonic coefficients by their degree, $l$, and not by their order, $m$ (see (2.13)), a sequence of uncoupled eigenproblems are obtained parameterized by the integer wavenumber $m \geq 0$. Due to the triangular truncation used (see $(2.7))$, the number of coefficients involved in the eigenvalue problem corresponding to the wavenumber $m$ is $3(L-m+1)$ if $m \geq 1$, and $3 L$ if $m=0$, because $\Psi_{0}^{0}=\Phi_{0}^{0}=0$ and (2.17) for $\Theta_{0}^{0}$ is not coupled to the rest and it is purely diffusive. Consequently it does not contribute to the instability.

Collocation methods have been used for the discretization of the radial operators. They were employed successfully by the authors in the case of spherical shells [23, 41, 21, 24, 20]. They are very efficient due to the possibility of using optimized matrix-matrix products to evaluate the action of the operators in order to compute the nonlinear terms in the time evolution codes of the full nonlinear equations. Moreover the simplicity of collocation methods allows one to easily change the boundary conditions. In this way each radial operator in (2.15)(2.17) is substituted by a matrix approximating it, associated to a given radial mesh, which in this work is of Gauss-Lobatto of $N+1$ points. The origin is included in the mesh, but the equations are only written at the inner points of the radial interval. The value of $\Phi_{l}^{m}$ and $\Theta_{l}^{m}$ at $r=1$ is zero, and that of $\Psi_{l}^{m}$ can be written in terms of the values at the inner points by using the boundary condition $\partial_{r}\left(\Psi_{l}^{m} / r\right)=0$. All the details can be found in [50]. A study of different meshes and ways of eliminating spurious eigenvalues are described there.

Since the operator $\mathcal{D}_{l}$ on the left-hand side of (2.16) is invertible, the eigenvalue problem (2.15)-(2.17) for a given azimuthal wavenumber $m$ can be written as a standard one,

$$
\mathcal{A}_{m}(\mathrm{Ra}, \mathrm{E}, \mathrm{Pr}) X_{m}=\lambda X_{m},
$$

$X_{m}=\left(\Psi_{m}, \Phi_{m}, \Theta_{m}\right)$ being a vector of dimension $3(L-m+1)(N-1)$ if $m \neq 0(3 L(N-1)$ if $m=0)$ containing the values of the amplitudes of the potentials and the perturbation of the temperature in spherical harmonics at the $N-1$ inner collocation points.

The matrices $\mathcal{A}_{m}$ are block-tridiagonal due to the structure of $\mathcal{Q}$, with $(L-m+1)$ if $m \neq 0(L$ if $m=0)$ rows of blocks of dimension $3(N-1)$. They are real only for $m=0$ and depend on the three nondimensional parameters. Since the problem is not self-adjoint the matrices $\mathcal{A}_{m}$ are not symmetric and the instability of the conduction state is almost always via a Hopf bifurcation. When the corresponding eigenfunction is no longer invariant under azimuthal translations, the transition gives rise, generically, to waves traveling in this direction [27]. This is the case when $m_{c} \neq 0, m_{c}$ being the critical azimuthal wavenumber for 
which $\Re(\lambda)=0$. If $m_{c}>0$ and $\lambda=i \omega_{c}$, the negative frequencies give rise to positive drifting velocities $c=-\omega_{c} / m_{c}$, i.e., the waves drift in the prograde direction. It was shown in [49] that if $m_{c}=0$, the bifurcation may break the reflection through the equator. The eigenfunction is antisymmetric, and the bifurcating oscillations are symmetric cycles, i.e., advancing half the period is equivalent to applying an equatorial reflection.

The initial leading spectra of $\mathcal{A}_{m}$ (defined as a set of eigenvalues of maximal real part) required to find initial conditions for the continuation of Hopf and double Hopf curves are computed by using shift-invert preconditioners. The eigenvalue problem $\mathcal{A}_{m} X_{m}=\lambda X_{m}$ is transformed into $\left(\mathcal{A}_{m}-\sigma \mathcal{I}\right)^{-1} X_{m}=\mu X_{m}$, where $\sigma$ is a complex shift. The eigenvectors of both problems are the same, and the eigenvalues are related by $\lambda=\sigma+1 / \mu$. Finding the $\mu$ 's of largest modulus is relatively easy using subspace iteration or Arnoldi algorithms [45]. The second option is employed here by means of the ARPACK package [39]. In this way the eigenvalues of $\mathcal{A}_{m}$ closest to $\sigma$ are found. A sequence of shifts of the form $\sigma=\rho+i \omega$ close to the imaginary axis is examined, using the information of nearby calculations, to ensure that no modes close to the critical are missed. The linear systems with matrix $\left(\mathcal{A}_{m}-\sigma I\right)$ are solved by means of an adapted LU decomposition making use of its block-tridiagonal structure. To avoid systems close to singular near the bifurcation points, when $\lambda=\lambda_{r}+i \lambda_{i}$ with $\lambda_{r} \approx 0$, a small real part $\rho \gtrsim 0$ is always included in the shift $\sigma$.

The critical values of the parameters are found by applying a root-finding algorithm to the equation $\lambda_{r}=0$ with two of the parameters fixed (usually E and Pr). For any set of parameters the truncation constants $N$ and $L$ have been taken to bound the errors in the critical parameters and frequencies below 1\%. In most of the computations in this article $N=50$ and $L=80$. This gives a maximum dimension for the complex matrices $\mathcal{A}_{m}$ of 11760. As will be seen in the next section, nonlinear systems of twice this size have to be solved. More detailed information on the methods used, and the results for wide intervals of the nondimensional parameters and of the wavenumber, ranging from $m=0$ to $m=60$, can be found in $[49,50]$.

3. Continuation of Hopf and double Hopf points. The curves of Hopf points of a given $m$ can be found by fixing one of the three parameters of the problem, increasing in small steps a second one, and solving the equation $\lambda_{r}=0$, with the first two parameters fixed, to find the critical third. If, in addition, the above-mentioned strategy of finding the spectra using a sequence of shifts is employed, the possibility of missing a bifurcation due to overtakings of eigenvalues (real parts growing at different rates when the parameters are moved) is minimized. Another way of tracing the curves is by fixing again one of the three parameters, and to apply a continuation technique to the extended nonlinear system of equations (see [29, 28])

$$
\begin{aligned}
\left(\mathcal{A}_{m}(\mathrm{Ra}, \mathrm{E}, \mathrm{Pr})-i \omega \mathcal{I}\right) X_{m} & =0, \\
\left\|X_{m}\right\|^{2} & =1, \\
\left\langle\Re\left(X_{m}\right), \Im\left(X_{m}\right)\right\rangle & =0,
\end{aligned}
$$

$\Re\left(X_{m}\right)$ and $\Im\left(X_{m}\right)$ being the real and imaginary parts of $X_{m}$, and $\langle\cdot, \cdot\rangle$ the Euclidean dot product. The second and third equations are normalizing conditions which uniquely determine the eigenvector $X_{m}$. Assume, for instance, that $\operatorname{Pr}$ is fixed. Then if the dimension of $\mathcal{A}_{m}$ is $n$, there are $2 n+2$ equations $\left(X_{m}\right.$ is complex) and $2 n+3$ unknowns $\left(X_{m}, \omega, \mathrm{Ra}, \mathrm{E}\right)$. This 
defines a curve of Hopf bifurcations. The continuation algorithm tracks the eigenfunction and associate frequency starting at a given initial condition. If there are overtakings of eigenvalues, the critical values of the parameters and frequency may not correspond to the first Hopf bifurcation. Therefore postprocessing is needed to validate the calculations, computing the leading spectra at the values of the parameters along the curve, but using the above strategy of sweeping shifts. This also has to be done in the case of curves of double Hopf points. In this latter case the extended system to track the simultaneous bifurcation to wavenumbers $m=m_{1}$ and $m=m_{2}$ is

$$
\begin{aligned}
\left(\mathcal{A}_{m_{1}}(\mathrm{Ra}, \mathrm{E}, \mathrm{Pr})-i \omega_{1} \mathcal{I}\right) X_{m_{1}} & =0, \\
\left(\mathcal{A}_{m_{2}}(\mathrm{Ra}, \mathrm{E}, \mathrm{Pr})-i \omega_{2} \mathcal{I}\right) X_{m_{2}} & =0, \\
\left\|X_{m_{1}}\right\|^{2} & =1, \\
\left\langle\Re\left(X_{m_{1}}\right), \Im\left(X_{m_{1}}\right)\right\rangle & =0, \\
\left\|X_{m_{2}}\right\|^{2} & =1, \\
\left\langle\Re\left(X_{m_{2}}\right), \Im\left(X_{m_{2}}\right)\right\rangle & =0 .
\end{aligned}
$$

Now, if the dimensions of $\mathcal{A}_{m_{1}}$ and $\mathcal{A}_{m_{2}}$ are $n_{1}$ and $n_{2}$, respectively, there are $2 n_{1}+2 n_{2}+4$ equations, and $2 n_{1}+2 n_{2}+5$ unknowns $\left(X_{m_{1}}, X_{m_{2}}, \omega_{1}, \omega_{2}, \mathrm{Ra}, \mathrm{E}, \mathrm{Pr}\right)$. All three parameters are obtained now during the continuation. It is clear from (3.4)-(3.9) that the minimal number of parameters in a system in order to have curves of this type of codimension-two bifurcations is three.

It must be said that the extended systems for Hopf and double Hopf points usually include the equations determining the equilibria of which the bifurcations are studied [29]. There is no need to add them in the present problem because the equilibrium is the trivial zero solution. Other extended systems to follow Hopf points are available (see [28]). They are based in reformulations of (3.1)-(3.3), or in using bialternate products. The latter method is not useful for large-scale systems because it implies processing huge nonsparse matrices.

During the continuation process Newton's method is used to solve the nonlinear systems. Inside this outer loop there is a second one since iterative methods have been used to solve the linear systems at each Newton step. Matrix-free methods are commonly used for this purpose. GMRES [47] has been the choice in this work. This combination leads to one of the possible Newton-Krylov methods. The name Krylov is associated to the use of linear solvers based on Krylov subspaces, GMRES being just one of them. A generic continuation code to track solutions of a nonlinear vector function requires subroutines to evaluate the function, the action of the Jacobian of the function on a vector, and a preconditioning one. The latter solves systems with a matrix approximating the Jacobian, enlarged with an additional row coming from the additional constraint required to select a single solution, and with a new column containing the derivatives of the function relative to the continuation parameter. Reference [51] is a recent tutorial on these techniques applied to the continuation of different invariant objects, not only equilibria. The theory corresponding to the case of the computation of the curves of double Hopf points has been included in the appendix.

In the present problem the computation of the left-hand sides of (3.4)-(3.9) and the action by their Jacobians is not difficult since the blocks of the matrices $\mathcal{A}_{m}$ can be easily computed. 
4. Validation of the double Hopf points. After computing the double Hopf curves, one must be sure that no other eigenvalues have crossed the imaginary axis, becoming the most unstable. Therefore the stability analysis of the conduction state is studied for the values of the parameters at each point of the double Hopf curves, and for several values of $m$ including those defining the double Hopf problem $\left(m_{1}\right.$ and $m_{2}$ in (3.4)-(3.9)). The method based on a sweep of complex shifts is used. A new Hopf bifurcation with $m=m_{3}$ indicates the presence of a triple Hopf point. When this happens, the curves corresponding to the double Hopf problems for $m_{1}-m_{3}$ and $m_{2}-m_{3}$ must be computed because they can form part of the boundary for the transition to $m=m_{1}$ or $m=m_{2}$. An example of this situation will be shown in the next section.

5. Results. The first step to track the double Hopf curves is finding the initial conditions. Figure 5.1(a) displays three curves of Hopf points given by (3.1)-(3.3) for $m=0$ and $m=1$. There are two curves in the latter case corresponding to azimuthal waves traveling eastwards or westwards relative to the frame of reference of the rotating sphere. This means that the frequency $\omega$ is negative and positive, respectively, as can be seen in Figure 5.1(b). The equivalent terms prograde (p) and retrograde (r) are used in the figure. The interval of $\mathrm{Pr}$ considered was based on the previous experience of the authors on the problem (see [49]). The conduction state is stable to perturbations of any wavenumber in the region indicated below the lower envelope of the Hopf curves of Figure 5.1(a). Their intersections are the initial conditions for the continuation of the double Hopf loci. The parameters corresponding to these points are given in Table 5.1. The first transition is then to axisymmetric solutions $(m=0)$ if $8.304 \times 10^{-3} \leq \operatorname{Pr} \leq 12.22 \times 10^{-3}$. By tracking the dependence of this interval with $\mathrm{E}$, the region in the parameter space $\mathrm{Pr}-\mathrm{E}$, for which the first bifurcation is to axisymmetric solutions, can be determined.

The curves of Figure 5.1(a) are the intersections of the surfaces of Hopf points shown in Figure 5.2(a) with the plane $\mathrm{E}=10^{-3}$. It contains, in addition, small segments of the double Hopf curves around this value of $\mathrm{E}$, and their projections onto the plane $\mathrm{Ra}=4 \times 10^{3}$. The
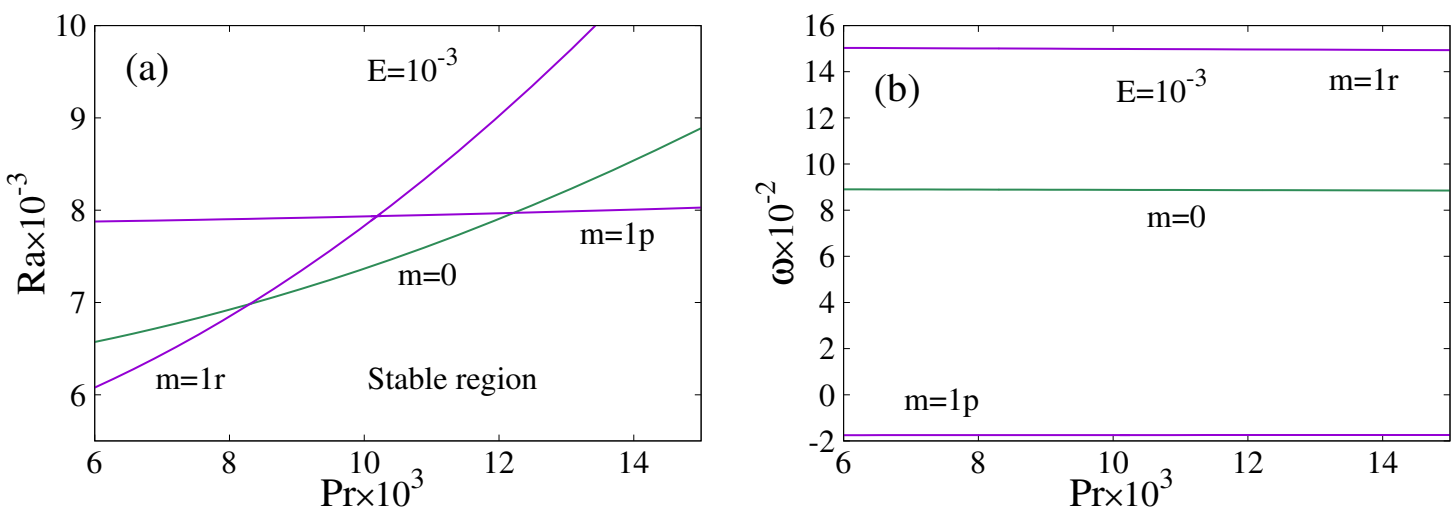

Figure 5.1. (a) Curves of Hopf points for $\mathrm{E}=10^{-3}$ for $m=0$ (green) and $m=1$ (violet). (b) The frequency, $\omega$, along the curves of Hopf points. The letters $p$ and $r$ mean prograde and retrograde drift, respectively. 
Table 5.1

Values of the parameters, wavenumbers, and frequencies at the initial double Hopf points.

\begin{tabular}{|c|c|c|c|c|r|r|}
\hline $\mathrm{E}$ & $\mathrm{Pr}$ & $\mathrm{Ra}$ & $m_{1}$ & $m_{2}$ & $\omega_{1}$ & $\omega_{2}$ \\
\hline $10^{-3}$ & $0.8304 \times 10^{-2}$ & 6983 & 0 & 1 & 889.0 & 1500.7 \\
\hline $10^{-3}$ & $0.1222 \times 10^{-1}$ & 7971 & 0 & 1 & 886.7 & -174.5 \\
\hline $10^{-3}$ & $0.1019 \times 10^{-1}$ & 7935 & 1 & 1 & 1498.7 & -174.7 \\
\hline
\end{tabular}
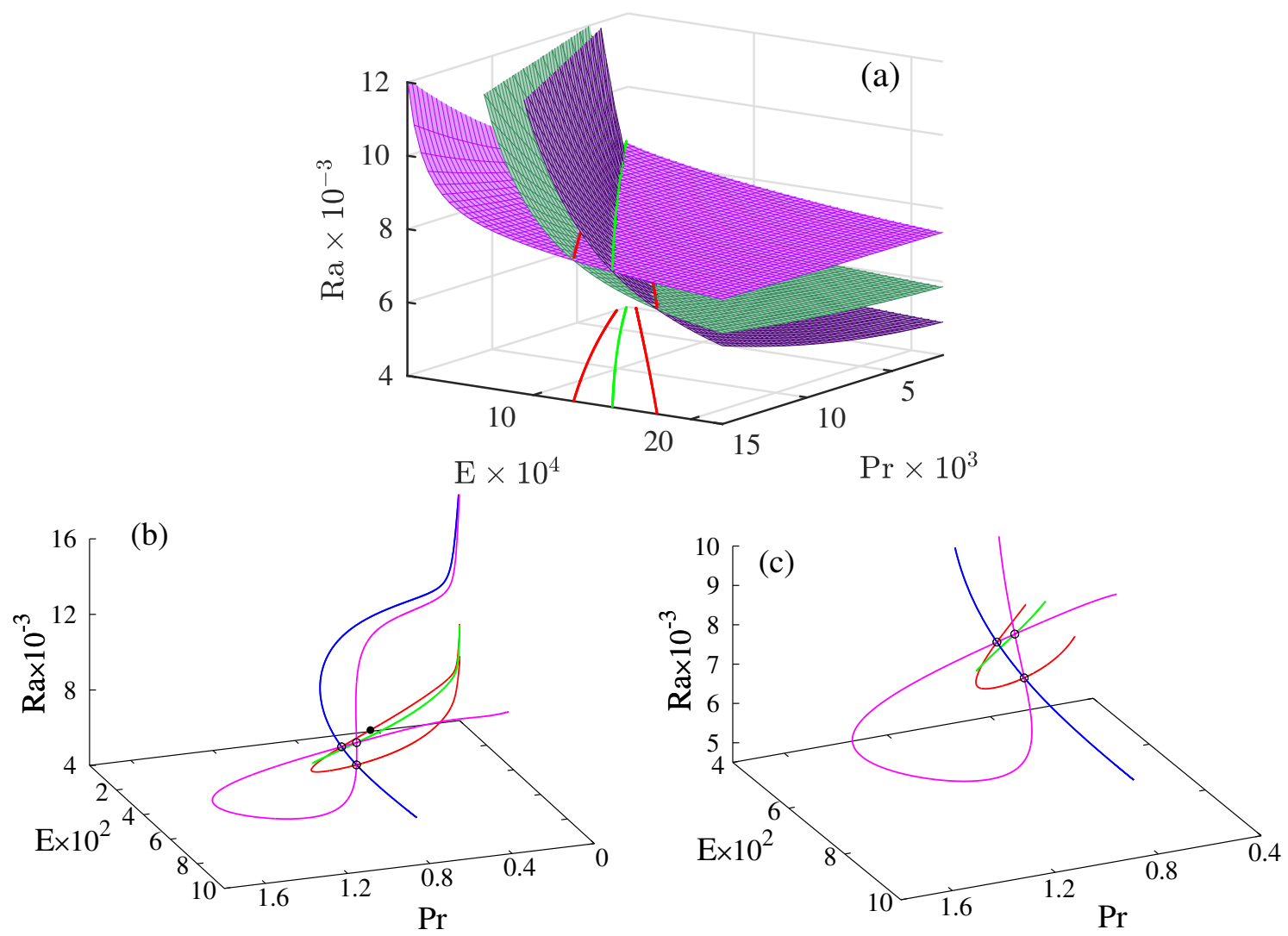

Figure 5.2. (a) Surfaces of Hopf points corresponding to $m=0$ (green), $m=1 r$ (dark violet), and $m=1 p$ (light violet) in the three-dimensional parameter space, together with a small portion of the double Hopf curves $m 01 p, m 01 r$ (red), and $m 1 p 1 r$ (green). The latter have also been projected onto the plane $\mathrm{Ra}=4 \times 10^{3}$. The conduction state is stable below the three surfaces. (b) Three-dimensional view of the full curves of double Hopf points. The blue and magenta curves correspond to the loci of double Hopf points for $m_{1}=0$ and $m_{2}=2$, and for $m_{1}=1$ and $m_{2}=2$, respectively. (c) Detail indicating the position of the triple Hopf points.

red curves correspond to the intersections of the critical surfaces $\left(\max _{\lambda} \Re(\lambda)=0\right)$ for $m_{1}=0$, and $m_{2}=1$ in the prograde and retrograde cases. They will be denoted by the symbols $m 01 p$ and $m 01 r$. The green one is the intersection of $m_{1}=1 p$ and $m_{2}=1 r$, denoted by $m 1 p 1 r$.

The full double Hopf loci are displayed in Figure 5.2(b), where it is shown that the curves $m 01 p$ and $m 01 r$ are in fact the same. At some point, indicated with a solid dot, the frequency 

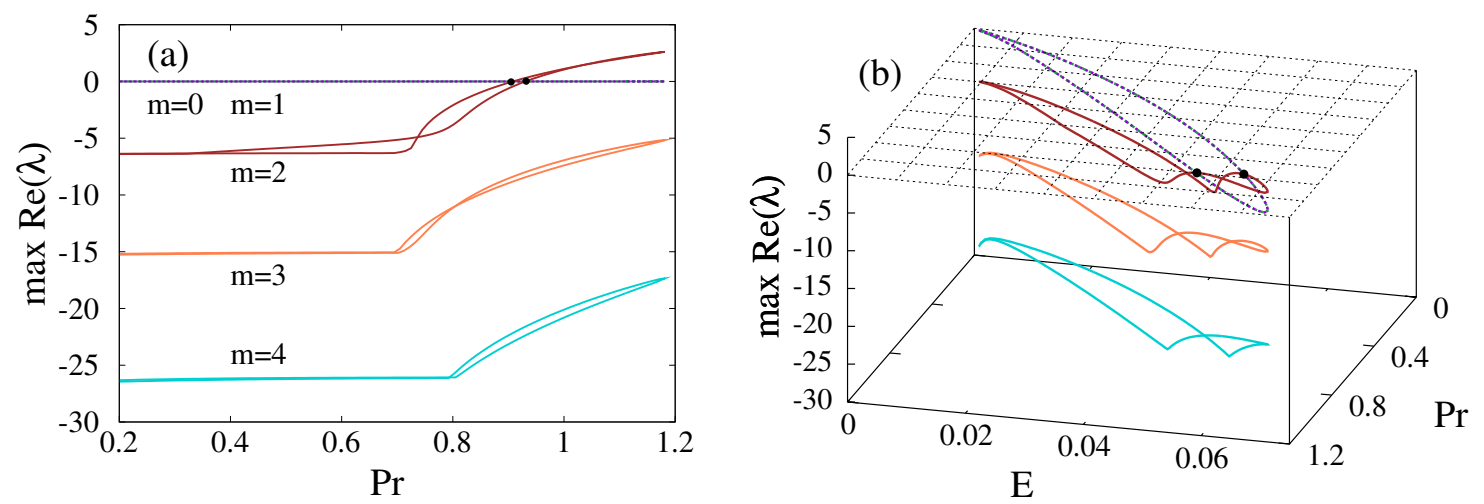

Figure 5.3. (a) Real part of the rightmost eigenvalue of the linear stability of the conduction state along the curve $m 01$, for $m=0,1,2,3,4$, as a function of $\mathrm{Pr}$. (b) Same results but as a function of the two parameters $\mathrm{E}$ and $\mathrm{Pr}$. The horizontal surface is $\max _{\lambda} \Re(\lambda)=0$.

$\omega$ changes sign on $m 01 p$, and the two curves merge there. The common curve will be denoted by $m 01$ from now on.

As explained in section 4 new Hopf bifurcations can occur along the double Hopf curves. This holds at the two empty dots on $m 01$ (red curve), shown in Figure 5.2(b), and in the detail of Figure 5.2(c). The meaning of the two other curves will be seen below. Figure 5.3 shows the real part of the rightmost eigenvalues of (2.15)-(2.17), for $m$ from 0 to 4 , along the curve $m 01$. The method of the sweep of shifts was used to obtain them. It can be seen that no eigenvalue corresponding to $m=0$ or $m=1$ crosses the imaginary axis along the curve $m 01$. The corresponding straight lines in Figures 5.3(a) and (b) are always superposed and constant to zero, indicating that the parameters correspond to critical values $(\Re(\lambda)=0)$. Moreover, the real part of all the eigenvalues corresponding to $m=3$ and $m=4$ is always negative along $m 01$. The same holds for $m=5$, but this is not presented here. The case $m=2$ is different; $\max _{\lambda} \Re(\lambda)$ crosses zero at two points, corresponding to the triple Hopf points just described. This was the method used to detect these codimension-three points. Two new curves of double Hopf points emerge there. The line drawn in blue in Figures 5.2(b) and (c) corresponds to $m_{1}=0$ and $m_{2}=2$, denoted from now on by $m 02$, and that in magenta to $m_{1}=1$ and $m=2$, and will be named $m 12$. The latter self-intersects at the third empty dot of Figures 5.2(b) and (c), which turns out to be on $m 1 p 1 r$.

When any of the curves tends to $(\mathrm{E}, \mathrm{Pr})=(0,0)$ the critical Ra tends to infinity (see Figure 5.2(b)). This limit is relevant in many astrophysical problems which have low $\mathrm{E}$ and Pr. The interval of the Ra axis has been limited for clarity. The curves $m 02$ and $m 1 p 1 r$ are double in this representation. They seem to end abruptly, but the endpoints are turning points. This can be seen in Figure 5.4. The frequencies $\omega_{1}$ and $\omega_{2}$ are plotted as functions of Pr and E along the four double Hopf curves. They have been separated for clarity. The plots for $m 01$ and $m 12$ are clear; there are two curves of frequencies. In Figure 5.4(b) there is only one because the role played by $\omega_{1}$ and $\omega_{2}$ and the corresponding eigenfunctions interchanges at the turning point, i.e., the curve is covered two times during the continuation. Finally, in Figure 5.4(c) there are two curves of frequencies. The curve corresponding to $m=0$ is 

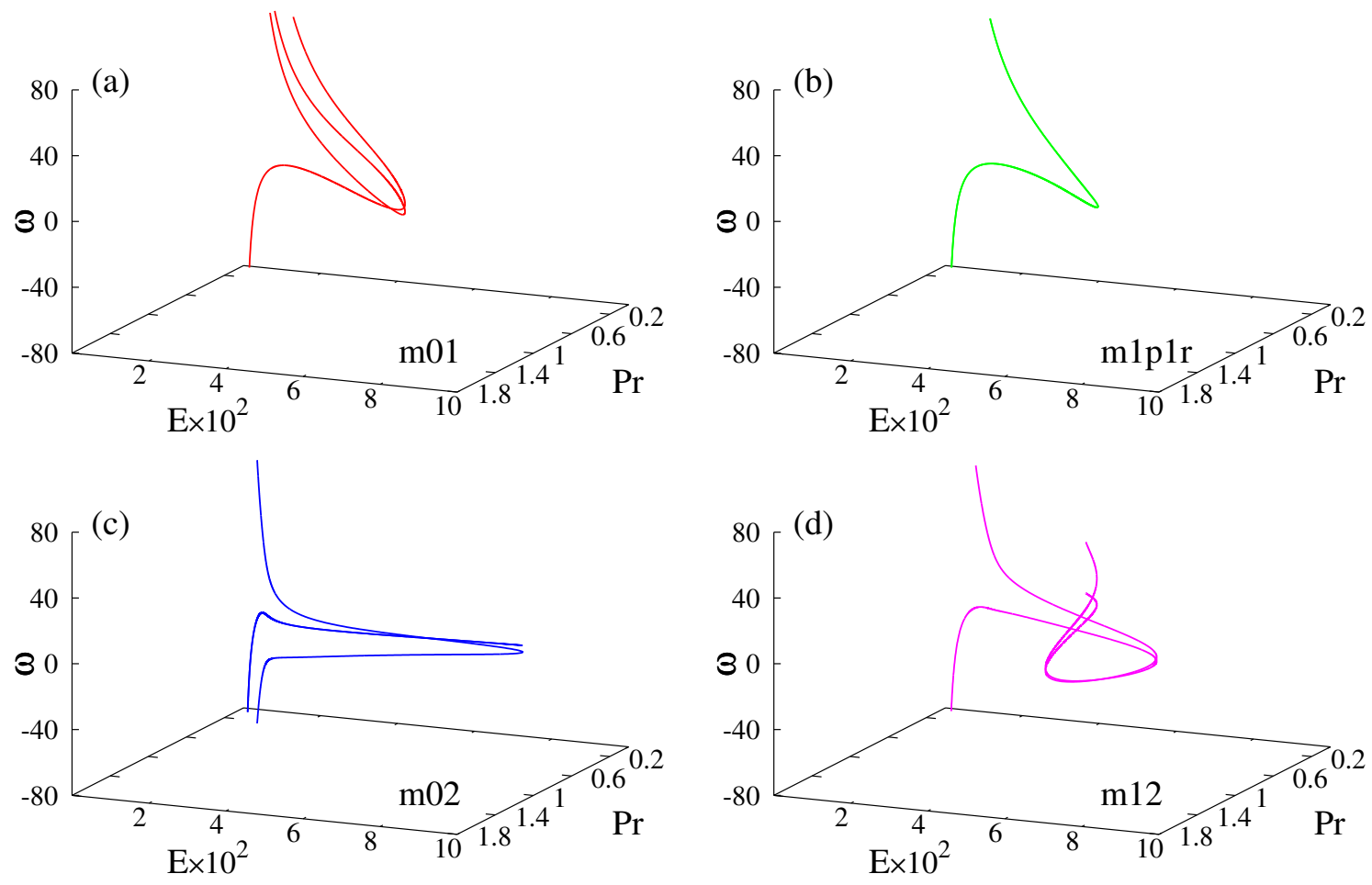

Figure 5.4. Frequencies along the double Hopf curves. The corresponding curve is indicated with a label in each plot.

symmetric with respect to the plane $\omega=0$. Its turning point occurs at $\omega=0$, and the curve continues with the conjugated eigenvalue and eigenfunction. Remember that in this case the eigenvalue problem is real. The second curve of frequencies, for $m=2$, just turns back, bouncing at the final point, and is covered two times, because if $\left(X_{m_{1}}, X_{m_{2}}, \omega_{1}, \omega_{2}, \operatorname{Ra}, \mathrm{E}, \mathrm{Pr}\right)$ is a solution of (3.4)-(3.9) with $m_{1}=0$, then so is $\left(\bar{X}_{m_{1}}, X_{m_{2}}, \bar{\omega}_{1}, \omega_{2}, \operatorname{Ra}, \mathrm{E}, \operatorname{Pr}\right)$. This happens every time the frequency corresponding to a real problem passes through zero.

Figure 5.5(a) shows the projection of the double Hopf curves of Figure 5.2(b) onto the parameter space Pr-E. The same information about the triple Hopf points and the point at which the frequency of the wavenumber $m=1$ changes sign on $m 01$ is contained in this figure. From all the previous information it is now clear that the region in the $\mathrm{Pr}-\mathrm{E}$ space in which the first bifurcation is to axisymmetric solutions, when Ra is increased from the stable zone, is limited by the curves $m 01$ and $m 02$. It has also been checked that the curve $m 1 p 1 r$ is never below the critical surface of $m=0$. The region has been plotted in Figure 5.5(c). Figure 5.5(b) is the same as Figure 5.5(a) but in logarithmic scale to show the behavior close to the origin. In the limit $\mathrm{Pr} \rightarrow 0$ the double Hopf curves follow linear laws of the form $\mathrm{E}=c\left(m_{1}, m_{2}\right) \operatorname{Pr}, c\left(m_{1}, m_{2}\right)$ being a constant depending on the wavenumbers defining the double Hopf problem. The values of $c\left(m_{1}, m_{2}\right)$ for the two branches of the curve $m 01, m 01 p$ and $m 01 r$ (in red in Figure 5.5(b)), are $c(0,1)=0.0744$ and 0.1326 , respectively. They were obtained by fitting the computed data in the interval $10^{-5} \leq \operatorname{Pr} \leq 10^{-3}$. This leaves, for 

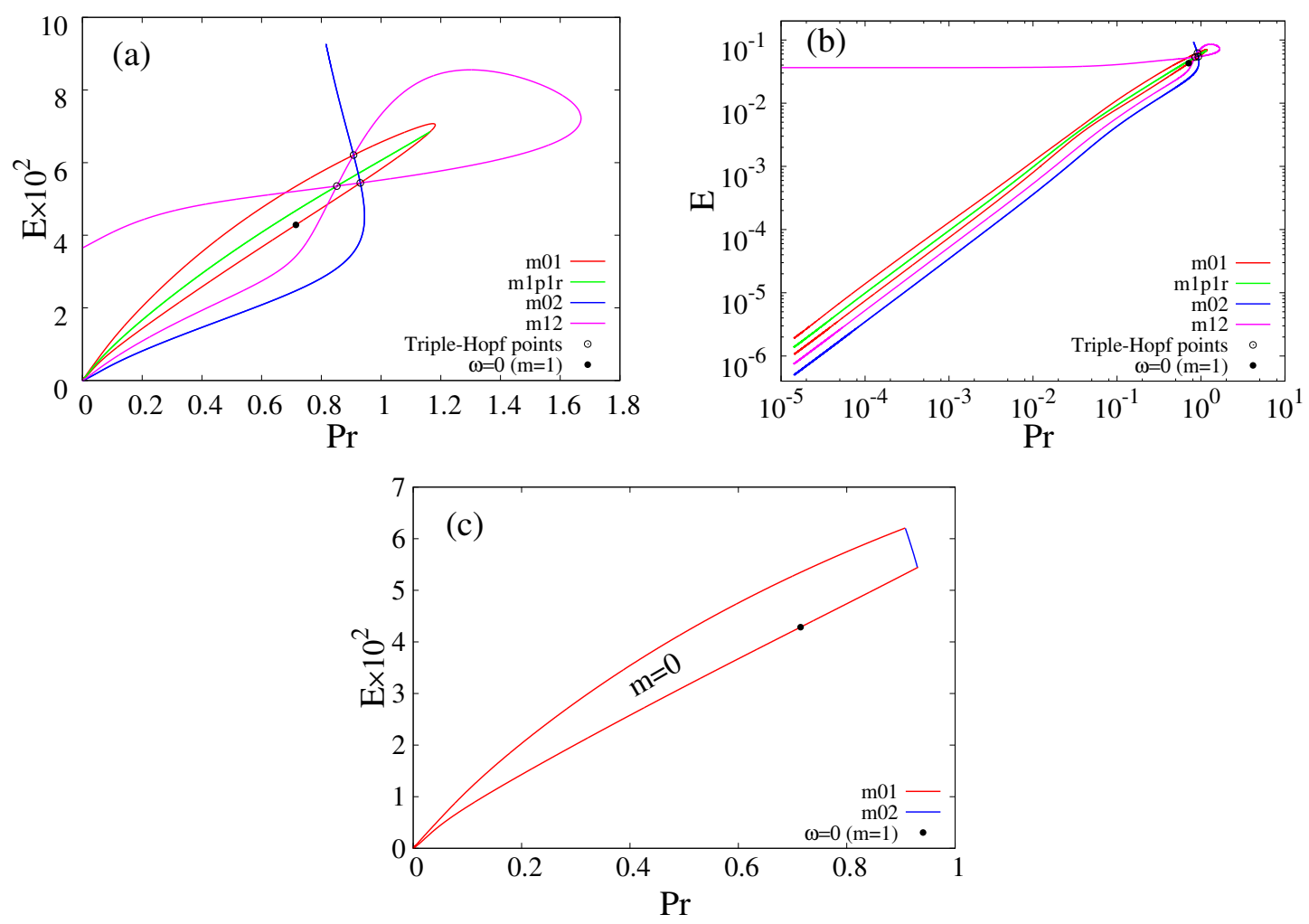

Figure 5.5. (a) Projection onto the plane Pr-E of the curves of Figure 5.2. The black dot indicates the point separating the $m=1$ prograde waves from those retrograde. (b) Same plot as (a) in logarithmic scale to show that for each value of $\mathrm{Pr}$ there is always an interval of $\mathrm{E}$ inside which the first bifurcation is to $m=0$. (c) Final region inside which the first bifurcation is to axisymmetric solutions.

each value of $\mathrm{Pr}$, an interval of $\mathrm{E}$ in which the first bifurcation is to $m=0$. The rest of the constants are $c(1 p, 1 r)=0.0971, c(0,2)=0.0346$, and $c(1,2)=0.0523$.

Figure $5.5(\mathrm{c})$ is relevant because it shows the range of parameters that must be used in nonlinear geophysical and astrophysical simulations to find oscillations of very high frequency, such as those necessary for the generation of magnetic fields, or oscillations able to produce strong zonal flows, such as those observed in the major planets. Although it can seem at first sight that the interval of stability is very narrow, the same happens with any azimuthal wave of any fixed azimuthal wavenumber $m \neq 0$. It is known that the nonlinear flows lose the axisymmetry of the eigenfunctions [37], but they retain most of the kinetic energy in their axisymmetric part ( $m=0$ modes). Although the analysis shown here does not determine the stability of the nonlinear solutions, it has been checked, using a three-dimensional temporal evolution code for a spherical shell of very small radius ratio and values of $\mathrm{Pr}$ and $\mathrm{E}$ contained in the $m=0$ region of Figure 5.5(c), that a nonperiodic torsional motion persists when Ra is increased.

Figures 5.6 to 5.8 show the eigenfunctions at the triple Hopf point located at $\operatorname{Pr}=0.9305$, $\mathrm{E}=5.441 \times 10^{-2}$, and $\mathrm{Ra}=1784$. Figure 5.6 corresponds to $m=0$. Four snapshots at 

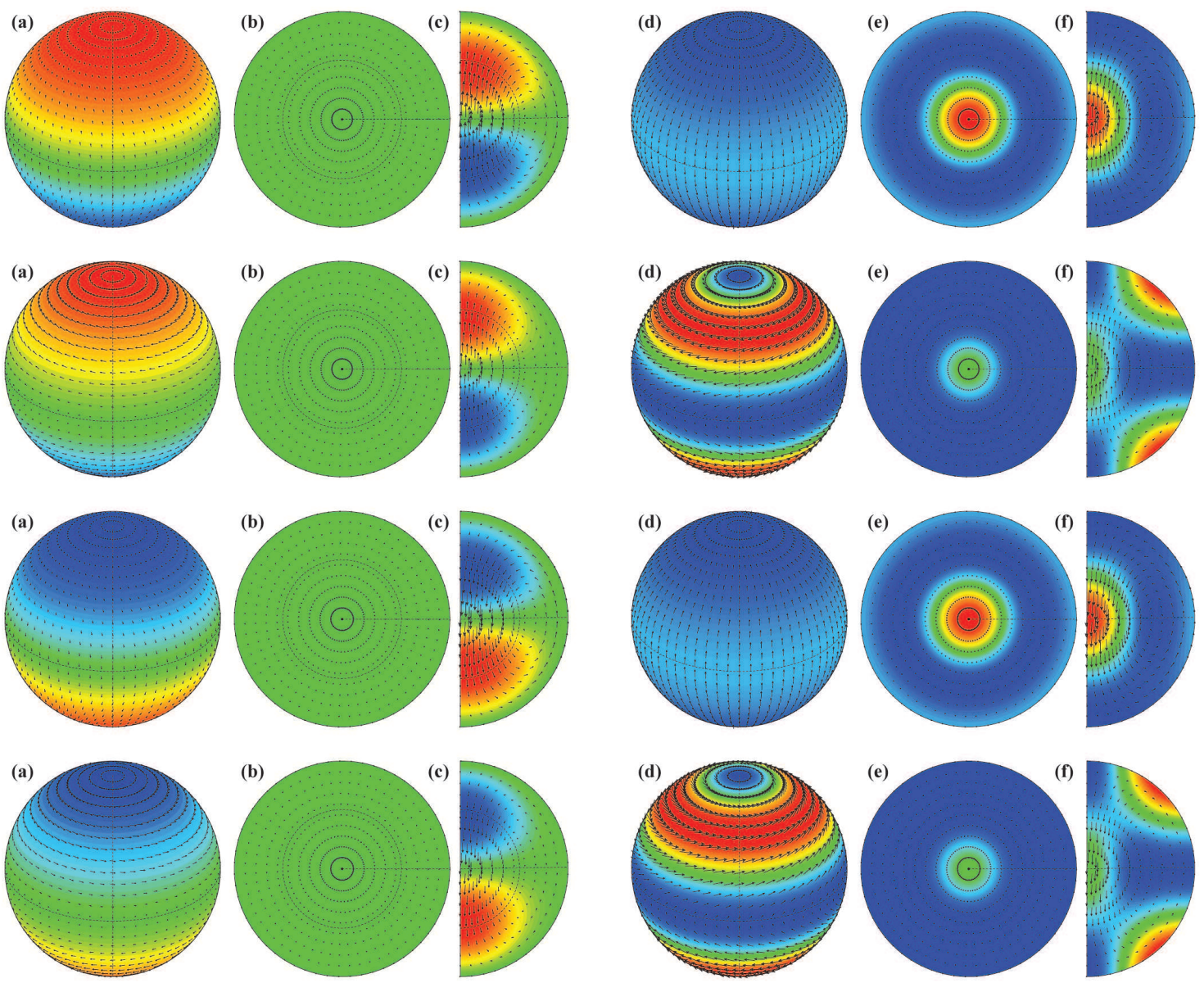

Figure 5.6. Contour plots of the perturbation of the temperature and velocity field (arrows) on (a) the spherical, (b) equatorial, and (c) meridional sections indicated by solid lines. (d), (e), and (f) show the same projections as (a), (b), and (c), but for the contour plots of the kinetic energy. The spherical section is taken at $r=0.5$ for the perturbation of the temperature and $r=1$ for the kinetic energy. The rows correspond, from top to bottom, to times $t=0, T / 4, T / 2$, and $3 T / 4, T$ being the period. The parameters are those of the triple Hopf point located at $\mathrm{Pr}=0.9305, \mathrm{E}=5.441 \times 10^{-2}$, and $\mathrm{Ra}=1784$. The azimuthal wavenumber is $m=0$.

times $t=0, T / 4, T / 2$, and $3 T / 4, T$ being the period, are presented. The spatiotemporal symmetry of the eigenfunction is quite obvious. Advancing half a period is equivalent to applying a reflection through the equator followed by a change of sign of the perturbation of the temperature, $\Theta$, and of the velocity field. In the first row, at $t=0$, the kinetic energy concentrates near the center, and most of it corresponds to the meridional velocity, the azimuthal one being small. At $t=T / 4$ there is an important zonal (azimuthal) flow, and the kinetic energy concentrates near the surface at mid latitudes. The other two rows can be obtained by the above-mentioned reflection with respect to the equator. More details of these axisymmetric eigenfunctions, and of the branches of torsional solutions emerging at this type of Hopf bifurcation, can be seen in [50, 53]. The temporal evolution for the other two eigenfunctions is much simpler; they are just rotating waves, so the patterns shown 

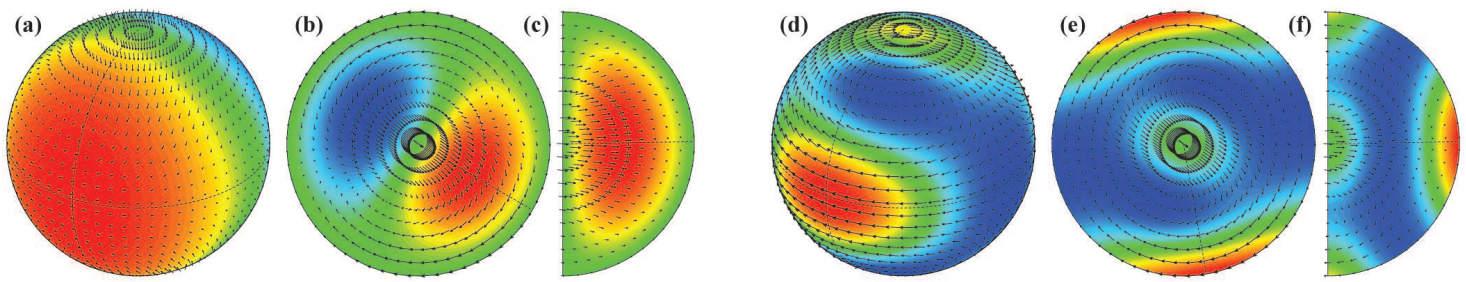

Figure 5.7. Same snapshots as in Figure 5.6 but for $m=1$. The spherical section is taken at $r=0.55$ for $\Theta$ and $r=1$ for the kinetic energy.
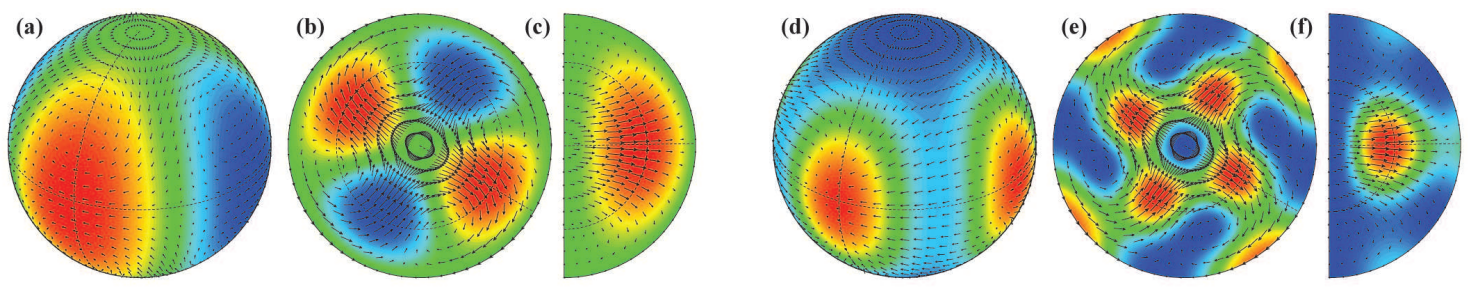

Figure 5.8. Same as Figure 5.7 but for $m=2$.

in Figures 5.7 and 5.8 just rotate as a rigid body around the vertical axis of the sphere. Movies showing the behavior of each of these cycles at this triple Hopf point have been included as supplementary material files: M133396_01.gif [local/web 9.74MB], M133396_02.gif [local/web 11.2MB], M133396_03.gif [local/web 8.82MB], M133396_04.gif [local/web 9.25MB], M133396_05.gif [local/web 12.4MB], and M133396_06.gif [local/web 10.4MB].

In order to understand how the double Hopf curves disappear at their turning points, and to have a better view of the critical surfaces, the continuation of the Hopf points for $m=0,1,2$ was performed for several fixed values of Pr, indicated by vertical lines in Figure 5.9(a). In all subsequent plots of Figures 5.9 and in Figures 5.10 and 5.11 the panels contain the critical curves (left) and the associated frequencies (right) for the Pr indicated in their labels. The intersections between curves of Hopf points, in each left plot, can be identified with the intersections of the corresponding vertical line with the double Hopf curves in Figure 5.9(a). The first three rows for $\operatorname{Pr}=0.80,0.85$, and 1.00 show the disappearance of the interval of first bifurcations to $m=0$ due to the displacement downwards of the $m=2$ curve. At $\operatorname{Pr} \approx 0.85$ there is a triple Hopf point, which is the intersection of the two $m=1$ branches with that of $m=2$. Between $\operatorname{Pr}=0.85$ and $\operatorname{Pr}=1.00$ the $m=2$ curve passes over the double Hopf points, which are intersections of the $m=0$ and $m=1$ curves, giving rise to the other two triple Hopf bifurcations.

The two $m=1$ curves for $\operatorname{Pr}=0.80,0.85$, and 1.00 in Figure 5.9 are in fact the same, as can be seen in Figures 5.10 and 5.11. They show how the self-intersection disappears between $\operatorname{Pr}=1.15$ and $\operatorname{Pr}=1.17$. It coincides with the final point of the green curve $m 1 p 1 r$ in Figure 5.9 (a). This can be confirmed by looking at the plots of the frequencies. At $\operatorname{Pr}=1.18$ the curve for $m=1$ is already univaluated, without turning points. At approximately this value of $\operatorname{Pr}$ the intersection of the curves $m=0$ and $m=1$ is double, indicating the turning point of the double Hopf curve $m 01$. 

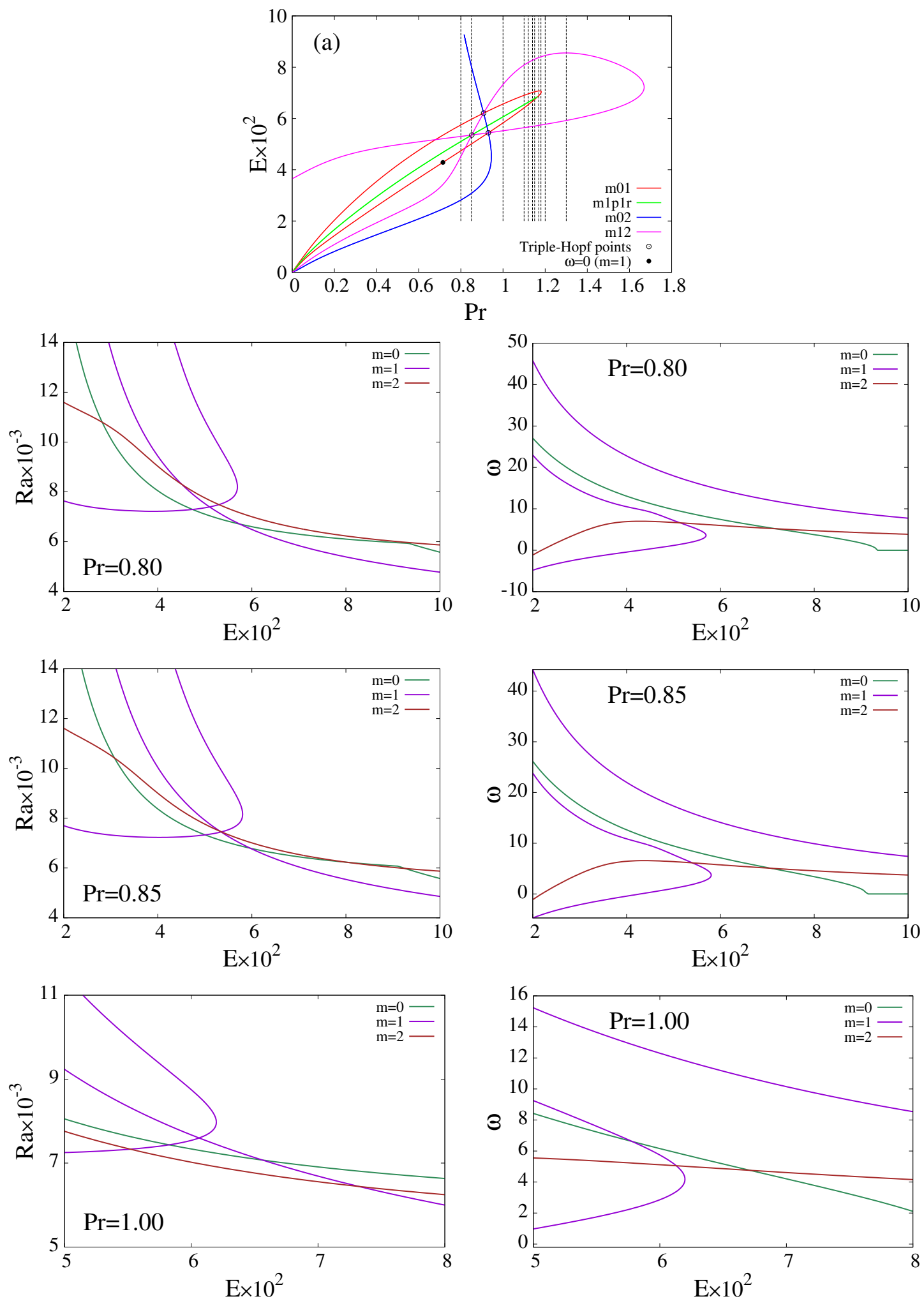

Figure 5.9. (a) Same as Figure 5.5(a), including vertical lines indicating the Pr of the plots of the rest of the panels of this figure, and of Figures 5.10 and 5.11. The remaining rows show curves of critical Ra (left) and frequencies (right) versus $\mathrm{E}$, corresponding to Hopf bifurcations for the wavenumbers $m=0,1,2$. The values of $\operatorname{Pr}$ are indicated in the labels. 

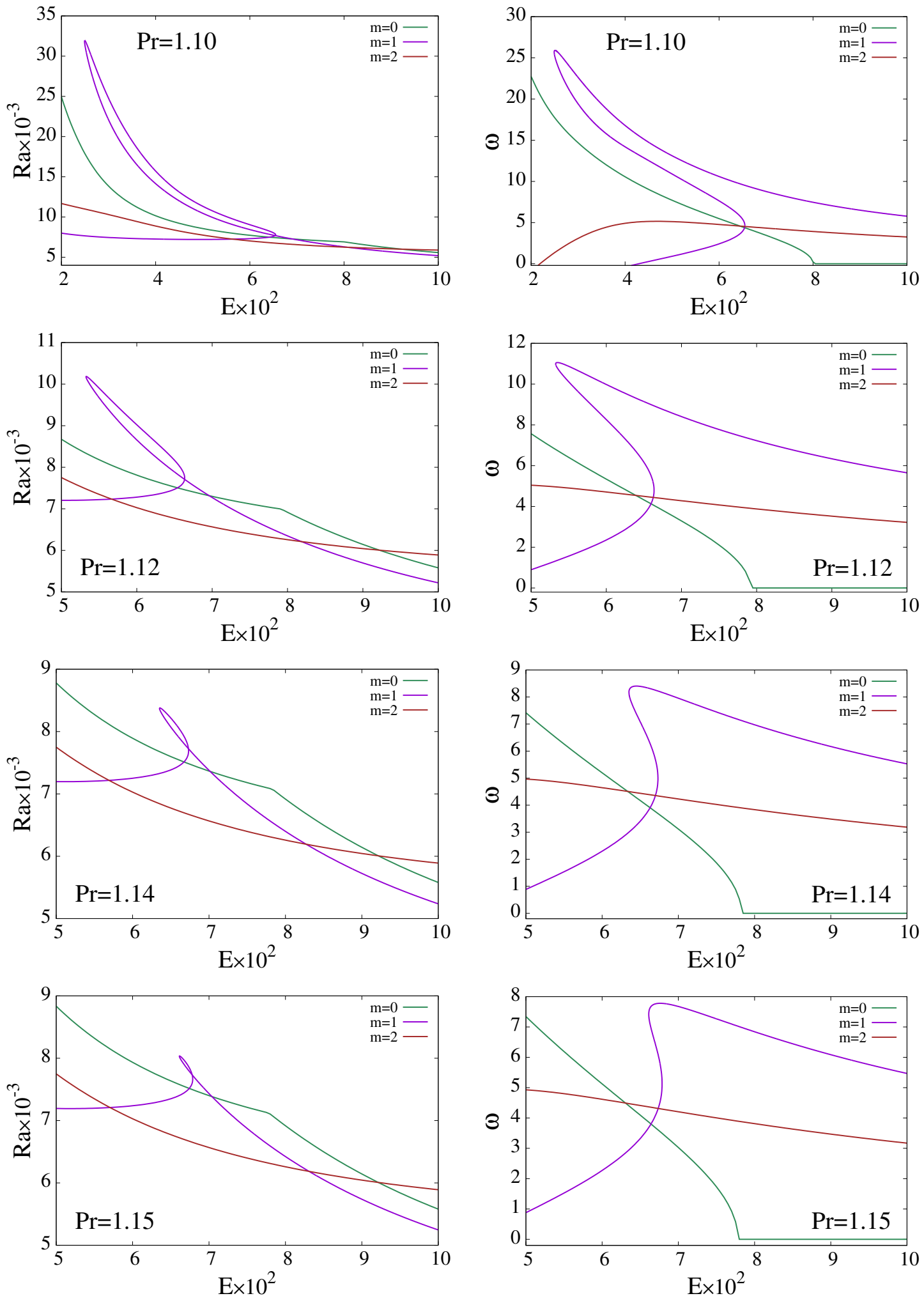

Figure 5.10. Same as last three rows of Figure 5.9 for the values of Pr indicated in the panels. 

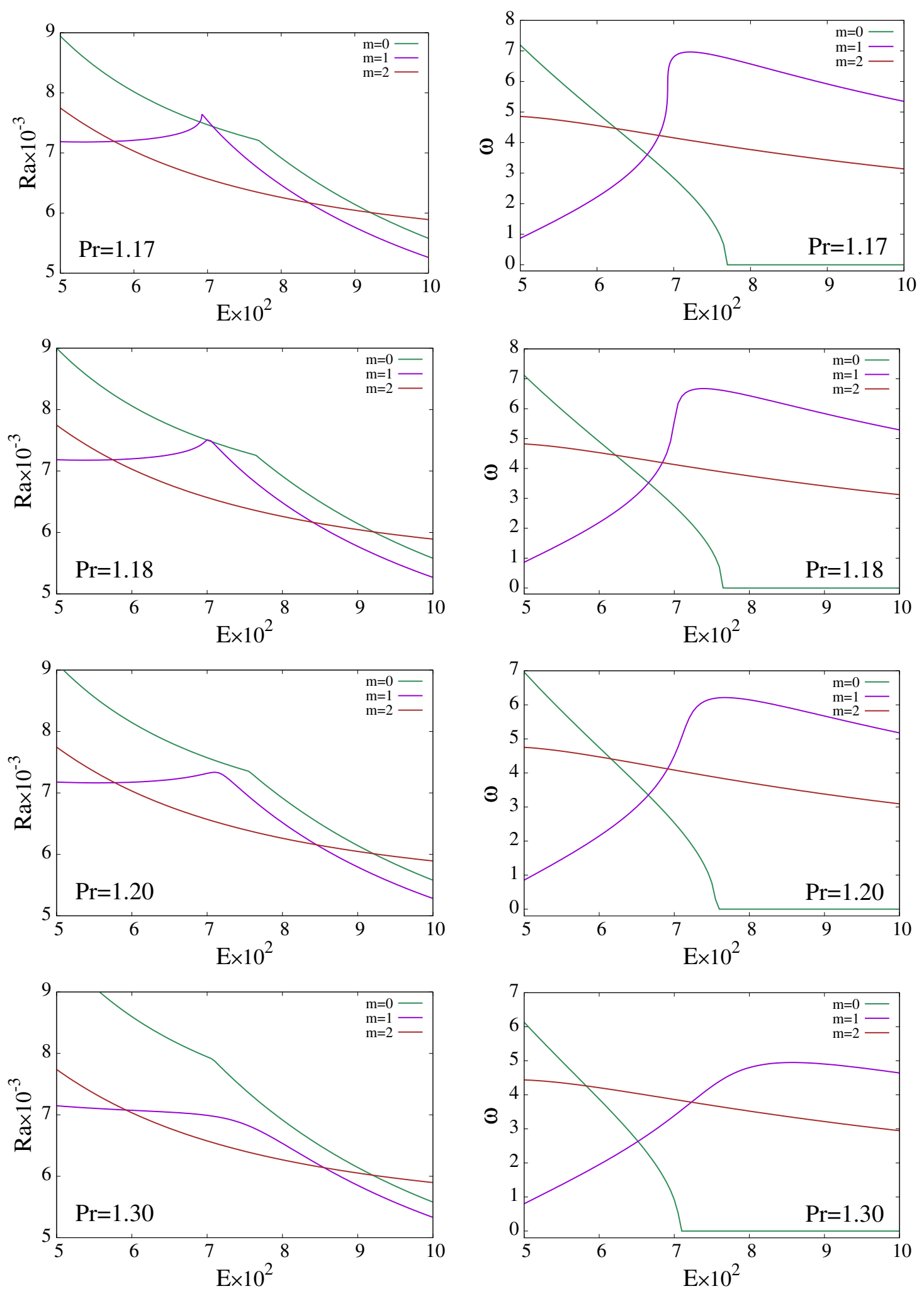

Figure 5.11. Same as Figure 5.10 for the values of Pr indicated in the panels. 
Each curve of bifurcation to solutions with $m=0$ is the envelope of two curves. The cusp points in the left plots of the critical Ra of Figures 5.10 and 5.11 mark the change. In the plots of the critical frequency, there is a positive frequency on the left part of the curves and a zero frequency on the right. The latter corresponds to a pitchfork bifurcation (not to a Hopf one, as in all the other cases). There is a curve of codimension-two Hopf-pitchfork points passing through the intersections of the curves labeled $m=0$ and $m=2$ in the plots of critical Ra at $\mathrm{E} \approx 9.2 \times 10^{-2}$. It has not been computed, since the pitchfork bifurcations never occur as the leading instability, at least for the range of parameters explored here.

6. Final remarks. The first objective of this work was to determine the region of the parameter space in which the first bifurcation is to solutions of azimuthal wavenumber $m=0$, and antisymmetric with respect to the equator, at least the region containing the interval previously obtained for low Ekman numbers [49]. The second goal was to display an algorithm for the continuation of double Hopf points in large-scale dissipative systems of discretized PDEs.

The mentioned region is bounded by curves of double Hopf points corresponding to pairs of wavenumbers $(0,1)$ and $(0,2)$. Their intersections correspond to codimension-three triple Hopf points. These results are summarized in Figure 5.5(c). The Prandtl numbers of the fluids of this figure include liquid metals of $\operatorname{Pr}<\mathcal{O}\left(10^{-1}\right)$, which are relevant for astrophysical and geophysical problems, but also gases of $\operatorname{Pr}=\mathcal{O}(1)$, at low rotation rates and Rayleigh numbers. Close to the origin, $(\operatorname{Pr}, \mathrm{E}) \approx(0,0)$, Ra rises quickly along the double Hopf curves. In addition, a detailed description of the way the boundary of the stability region is reached has been given.

The torsional modes described here were found while exploring the linear stability of the conduction state of a rotating fluid sphere. It would be interesting to explore in detail what happens in a spherical shell, and how the bifurcation diagram changes with the radius ratio. In this case the first bifurcation, or a bifurcation on the branch of periodic torsional flows could be subcritical, giving rise to stable velocity fields of complex time dependence at very low Ra. These fields are good candidates for the generation of strong magnetic fields such as those found in [52].

The numerical methods used are based on the matrix-free continuation of the solutions of adequate extended systems. This methodology is general and can be applied to any stability problem of systems governed by parabolic-elliptic PDEs. For instance, the regions of transitions to the well-known rotating waves, with a fixed nonzero azimuthal wavenumber, can also be determined with the same techniques. Although these methods are not new for low-dimensional systems of ODEs, their application to PDEs is not common at all, and only a few examples can be found in the literature, most of which are mentioned in the introduction.

Appendix A. Some details on the numerical methods used to track the curves of double Hopf points are given here. The case of Hopf points is simpler and can be deduced from that of the double Hopf bifurcations. The general framework of continuation methods for large-scale systems is explained in [51]. A minimal part is reproduced here for completeness, adding what is necessary for the particular systems arising in this article.

Consider a system of nonlinear equations depending on a parameter $p$,

$$
H(y, p)=0, \quad(y, p) \in \mathcal{U} \subset \mathbb{R}^{K} \times \mathbb{R},
$$


for which one is interested in its solutions and their dependence on $p$. In our case the system is that given by (3.4)-(3.9) with $y=\left(X_{m_{1}}, X_{m_{2}}, \omega_{1}, \omega_{2}, \mathrm{Ra}, \mathrm{E}\right), K=2 n_{1}+2 n_{2}+4$, and $p=\operatorname{Pr}$. It could be any of the other two parameters, Ra or E. Then Pr would be included in the vector $y$. Since the bifurcation curves are parameterized by the arclength it is not important which one is the distinguished parameter.

The curves, $(y(s), p(s))$, of solutions of (A.1), $s$ being the arclength, are traced by using continuation methods. They consist in two stages: a predictor step, in which an initial approximation to a new point on the curve $\left(y_{0}, p_{0}\right)$ is computed by extrapolation from the previously obtained solutions, and a corrector step, in which the prediction is refined by Newton's method. The most common continuation methods, parameter, local parameterization, and pseudo-arclength, admit a unified formulation by adding to (A.1) an equation,

$$
h(y, p)=0
$$

to determine simultaneously unique $y$ and $p$. If $h(y, p)=p-p_{0}$, the equation fixes the parameter $p$, leading to parameter continuation, which is the easiest method, but fails at turning points. If $h(y, p)$ is one of the components of $y$ or $p$, depending on which is growing faster, the method is described as a local parameterization method. Finally, $h(y, p)=h_{y}^{\top}(y-$ $\left.x_{0}\right)+h_{p}\left(p-p_{0}\right)$, where $\left(y_{0}, p_{0}\right)$ and $\left(h_{y}, h_{p}\right)$ are the predictions of a new point and the tangent to the curve of solutions, respectively, defines a pseudo-arclength method. In the two latter cases the hyperplane $h(y, p)=0$ will cut the curve transversely if the prediction is not far from the previous point, and the algorithm allows passing folds. In any case the system that determines a unique solution, $(y, p) \in \mathbb{R}^{K+1}$, is

$$
\widetilde{H}(y, p)=\left(\begin{array}{c}
H(y, p) \\
h(y, p)
\end{array}\right)=0 .
$$

Large-scale systems, $\widetilde{H}(y, p)=0$, are usually solved by inexact Newton methods. Instead of solving the linear systems by means of direct algorithms, iterative methods are used. If the linear solvers are based on Krylov subspaces (GMRES, BiCGStab, FOM, TFQMR, etc.; see, for instance, [46]) they are also called Newton-Krylov methods. In inexact Newton methods, a sequence of approximations are obtained, starting from the initial prediction $\left(y_{0}, p_{0}\right)$, with

$$
\left(y_{i+1}, p_{i+1}\right)=\left(y_{i}, p_{i}\right)+\left(\Delta y_{i}, \Delta p_{i}\right)
$$

where $\left(\Delta y_{i}, \Delta y_{i}\right)$ satisfies the linear system

$$
\left(\begin{array}{cc}
D_{y} H\left(y_{i}, p_{i}\right) & D_{p} H\left(y_{i}, p_{i}\right) \\
h_{y}^{\top} & h_{p}
\end{array}\right)\left(\begin{array}{c}
\Delta y_{i} \\
\Delta p_{i}
\end{array}\right)=\left(\begin{array}{c}
-H\left(y_{i}, p_{i}\right) \\
-h\left(y_{i}, p_{i}\right)
\end{array}\right),
$$

which is solved iteratively. The term matrix-free method is used when the linear solver only requires the user to provide matrix products. In our case the products have the form

$$
\left(\begin{array}{cc}
D_{y} H\left(y_{i}, p_{i}\right) & D_{p} H\left(y_{i}, p_{i}\right) \\
h_{y}^{\top} & h_{p}
\end{array}\right)\left(\begin{array}{c}
\delta y \\
\delta p
\end{array}\right)
$$


with $(\delta y, \delta p)^{\top}$ an arbitrary vector provided to the user by the linear solver. The linear solvers are not, in general, convergent for arbitrary systems, especially in the case of equilibria of discretizations of systems of parabolic PDEs. It is necessary, then, to use preconditioners to improve the convergence. This means a matrix approximating that of (A.3), and such that it is possible to solve systems with it in an efficient way. A common trick is to first approximate the matrix of (A.3) by

$$
\left(\begin{array}{cc}
D_{y} H\left(y_{i}, p_{i}\right) & 0 \\
0 & 1
\end{array}\right)
$$

which is just changing a row and column, and then trying to precondition just $D_{y} H\left(y_{i}, p_{i}\right)$.

The Jacobian on the left-hand side of (3.4)-(3.9) at $y=\left(X_{m_{1}}, X_{m_{2}}, \omega_{1}, \omega_{2}, \mathrm{Ra}, \mathrm{E}\right)$ has the form

$$
D_{y} H(y, p)=\left(\begin{array}{cccccc}
\mathcal{A}_{m_{1}}-i \omega_{1} \mathcal{I} & 0 & -i X_{m_{1}} & 0 & \partial_{\mathrm{Ra}} \mathcal{A}_{m_{1}} X_{m_{1}} & \partial_{\mathrm{E}} \mathcal{A}_{m_{1}} X_{m_{1}} \\
0 & \mathcal{A}_{m_{2}}-i \omega_{2} \mathcal{I} & 0 & -i X_{m_{2}} & \partial_{\mathrm{Ra}} \mathcal{A}_{m_{2}} X_{m_{2}} & \partial_{\mathrm{E}} \mathcal{A}_{m_{2}} X_{m_{2}} \\
\nabla N_{1} & 0 & 0 & 0 & 0 & 0 \\
\nabla N_{2} & 0 & 0 & 0 & 0 & 0 \\
0 & \nabla N_{3} & 0 & 0 & 0 & 0 \\
0 & \nabla N_{4} & 0 & 0 & 0 & 0
\end{array}\right),
$$

where, for simplicity, the dependence of the matrices $\mathcal{A}_{m}$ and their derivatives on Ra, E, and $\operatorname{Pr}$, and the dependence of the gradients of the four normalizing conditions (3.6)-(3.9), $N_{i}$, on $X_{m_{1}}$ or $X_{m_{2}}$ is not made explicit. Matrix products to vectors of the form $\delta y=$ $\left(\delta X_{m_{1}}, \delta X_{m_{2}}, \delta \omega_{1}, \delta \omega_{2}, \delta \mathrm{Ra}, \delta \mathrm{E}\right)$ have to be computed during the linear solving by matrix-free methods.

The preconditioning matrix used is

$$
\left(\begin{array}{cccccc}
\mathcal{A}_{m_{1}}-\left(\rho+i \omega_{1}\right) \mathcal{I} & 0 & 0 & 0 & 0 & 0 \\
0 & \mathcal{A}_{m_{2}}-\left(\rho+i \omega_{2}\right) \mathcal{I} & 0 & 0 & 0 & 0 \\
0 & 0 & 1 & 0 & 0 & 0 \\
0 & 0 & 0 & 1 & 0 & 0 \\
0 & 0 & 0 & 0 & 1 & 0 \\
0 & 0 & 0 & 0 & 0 & 1
\end{array}\right)
$$

which differs from (A.4) in the last four rows and columns, and in the perturbation of the frequencies $\omega_{k}$. The additional real part $\rho$ is added, as stated before, to avoid singular or close to singular systems when $i \omega_{k}$ is a good approximation of an eigenvalue of $\mathcal{A}_{m_{k}}$. Since the blocks of these matrices can be computed, the LU decomposition of the block-tridiagonal matrices $\mathcal{A}_{m_{k}}-\left(\rho+i \omega_{k}\right) \mathcal{I}$ is available, and solving systems with the matrix (A.5) is easy.

\section{REFERENCES}

[1] F. Al-Shamali, M. Heimpel, and J. Aurnou, Varying the spherical shell geometry in rotating thermal convection, Geophys. Astrophys. Fluid Dyn., 98 (2004), pp. 153-169. 
[2] J. M. Aurnou, Planetary core dynamics and convective heat transfer scaling, Geophys. Astrophys. Fluid Dyn., 101 (2007), pp. 327-345.

[3] A. P. Bassom, A. M. Soward, and S. V. Starchenko, The onset of strongly localized thermal convection in rotating spherical shells, J. Fluid Mech., 689 (2011), pp. 376-416.

[4] F. H. Busse, Thermal instabilities in rapidly rotating systems, J. Fluid Mech., 44 (1970), pp. 441-460.

[5] F. H. Busse, Convective flows in rapidly rotating spheres and their dynamo action, Phys. Fluids, 14 (2002), pp. 1301-1313.

[6] P. CARDin AND P. Olson, Chaotic thermal convection in a rapidly rotating spherical shell: Consequences for flow in the outer core, Phys. Earth Planet. Inter., 82 (1994), pp. 235-259.

[7] C. R. Carrigan And F. H. Busse, An experimental and theoretical investigation of the onset of convection in rotating spherical shells, J. Fluid Mech., 126 (1983), pp. 287-305.

[8] S. Chandrasekhar, Hydrodynamic and Hydromagnetic Stability, Oxford University Press, New York, 1961.

[9] U. Christensen, Zonal flow driven by strongly supercritical convection in rotating spherical shells, J. Fluid Mech., 470 (2002), pp. 115-133.

[10] U. Christensen And J. Aubert, Scaling properties of convection-driven dynamos in rotating spherical shells and application to planetary magnetic fields, Geophys. J. Int., 166 (2006), pp. 97-114.

[11] K. A. Cliffe, Numerical calculations of the primary-flow exchange process in the Taylor problem, J. Fluid Mech., 197 (1988), pp. 57-79.

[12] W. O. Criminale, T. L. Jackson, And R. D. Joslin, Theory and Computation in Hydrodynamic Stability, 2nd ed., Cambridge Monogr. Mech., Cambridge University Press, Cambridge, UK, 2018.

[13] H. A. Dijkstra, F. W. Wubs, A. K. Cliffe, E. Doedel, I. F. Dragomirescu, B. Eckhardt, A. Gelfgat, A. Hazel, V. Lucarini, A. Salinger, J. Sánchez, H. Schuttelaars, L. TuckERMAN, AND U. ThiEle, Numerical bifurcation methods and their application to fluid dynamics: Analysis beyond simulation, Commun. Comput. Phys., 15 (2014), pp. 1-45.

[14] N. Dinar And H. B. Keller, Computation of Taylor vortex flows using multigrid continuation methods, in Recent Advances in Computational Fluid Dynamics, C. C. Chao, S. A. Orszag, and W. Shyy, eds., Lecture Notes in Engrg. 43, Springer, 1989, pp. 191-262.

[15] E. Doedel, AUTO: Software for Continuation and Bifurcation Problems in Ordinary Differential Equations, Tech. report, California Institute of Technology, Pasadena CA, 1986.

[16] E. Doedel And L. S. Tuckerman, eds., Numerical Methods for Bifurcation Problems and Large-Scale Dynamical Systems, IMA Vol. Math. Appl. 119, Springer-Verlag, 2000.

[17] E. Dormy, A. M. Soward, C. A. Jones, D. Jault, And P. Cardin, The onset of thermal convection in rotating spherical shells, J. Fluid Mech., 501 (2004), pp. 43-70.

[18] P. Drazin And W. ReId, Hydrodynamic Stability, Cambridge Monographs on Mechanics, Cambridge University Press, Cambridge, UK, 1981.

[19] B. Favier And E. KNOBloch, Robust wall states in rapidly rotating Rayleigh-Bénard convection, J. Fluid Mech., 895 (2020), R1.

[20] F. Garcia, L. Bonaventura, M. Net, And J. SÁnchez, Exponential versus IMEX high-order time integrators for thermal convection in rotating spherical shells, J. Comput. Phys., 264 (2014), pp. 41-54.

[21] F. Garcia, M. Net, B. García-Archilla, and J. SÁnchez, A comparison of high-order time integrators for the Boussinesq Navier-Stokes equations in rotating spherical shells, J. Comput. Phys., 229 (2010), pp. 7997-8010.

[22] F. Garcia, J. SÁnchez, E. Dormy, And M. Net, Oscillatory convection in rotating spherical shells: Low Prandtl number and non-slip boundary conditions, SIAM J. Appl. Dyn. Syst., 14 (2015), pp. 17871807, https://doi.org/10.1137/15M100729X.

[23] F. Garcia, J. SÁnchez, And M. Net, Antisymmetric polar modes of thermal convection in rotating spherical fluid shells at high Taylor numbers, Phys. Rev. Lett., 101 (2008), 194501.

[24] F. Garcia, J. SÁnchez, AND M. NET, Numerical simulations of high-Rayleigh-number convection in rotating spherical shells under laboratory conditions, Phys. Earth Planet. Inter., 230 (2014), pp. 2844.

[25] A. Gelfgat, Computational Modelling of Bifurcations and Instabilities in Fluid Dynamics, Comput. Methods Appl. Sci. 50, Springer, Cham, 2018.

[26] N. Gillet, D. Brito, D. Jault, And H.-C. Nataf, Experimental and numerical studies of convection 
in a rapidly rotating spherical shell, J. Fluid Mech., 580 (2007), pp. 83-121.

[27] M. Golubitsky, I. Stewart, And D. G. Schaeffer, Singularities and Groups in Bifurcation Theory, Vol. II, Springer, New York, 1988.

[28] W. J. F. GovaerTs, Numerical Methods for Bifurcations of Dynamical Equilibria, SIAM, Philadelphia, 2000, https://doi.org/10.1137/1.9780898719543.

[29] A. Griewank and G. Reddien, The calculation of Hopf points by a direct method, IMA J. Numer. Anal., 3 (1983), pp. 295-303.

[30] B. W. Hindman, N. A. Featherstone, and K. Julien, Morphological classification of the convective regimes in rotating stars, Astrophys. J., 898 (2020), 120.

[31] C. A. Jones, A. M. Soward, And A. I. Mussa, The onset of thermal convection in a rapidly rotating sphere, J. Fluid Mech., 405 (2000), pp. 157-179.

[32] D. D. Joseph, Stability of Fluid Motions, Vol. I, Springer, 1976.

[33] D. D. Joseph, Stability of Fluid Motions, Vol. II, Springer, 1976.

[34] G. Kawahara, M. Uhlmann, and L. Van Veen, The significance of simple invariant solutions in turbulent flows, Ann. Rev. Fluid Mech., 44 (2012), pp. 203-225.

[35] H. B. Keller, Numerical solution of bifurcation and nonlinear eigenvalue problems, in Applications of Bifurcation Theory, P. H. Rabinowitz, ed., Academic Press, New York, 1977, pp. 359-384.

[36] I. Kitiashvili and A. G. Kosovichev, Application of data assimilation method for predicting solar cycles, Astrophys. J., 688 (2008), pp. L49-L52.

[37] D. Kong, K. Zhang, K. LAm, And A. P. Willis, Axially symmetric and latitudinally propagating nonlinear patterns in rotating spherical convection, Phys. Rev. E, 98 (2018), 031101.

[38] M. KubíčEK, Algorithm 502: Dependence of solution of nonlinear systems on a parameter, ACM Trans. Math. Softw., 2 (1976), pp. 98-107.

[39] R. B. Lehoucq AND D. C. Sorensen, Deflation techniques for an implicitly restarted Arnoldi iteration, SIAM J. Matrix Anal. Appl., 17 (1996), pp. 789-821, https://doi.org/10.1137/S0895479895281484.

[40] R. Meyer-Spasche AND H. B. Keller, Computation of the axisymmetric flow between rotating cylinders, J. Comput. Phys., 35 (1980), pp. 100-109.

[41] M. Net, F. Garcia, AND J. SÁnchez, On the onset of low-Prandtl-number convection in rotating spherical shells: Non-slip boundary conditions, J. Fluid Mech., 601 (2008), pp. 317-337.

[42] M. Net, F. Garcia, And J. SÁnchez, Numerical study of the onset of thermosolutal convection in rotating spherical shells, Phys. Fluids, 24 (2012), 064101.

[43] M. Net AND J. SÁnchez, Continuation of bifurcations of periodic orbits for large-scale systems, SIAM J. Appl. Dyn. Syst., 14 (2015), pp. 674-698, https://doi.org/10.1137/140981010.

[44] P. H. Roberts, On the thermal instability of a rotating fluid sphere containing heat sources, Phil. Trans. R. Soc. Lond. A, 263 (1968), pp. 93-117.

[45] Y. SAAD, Numerical Methods for Large Eigenvalue Problems, Manchester University Press, Manchester, UK, 1992.

[46] Y. SAAD, Iterative Methods for Sparse Linear Systems, PWS, New York, 1996.

[47] Y. SAAD AND M. H. Schultz, GMRES: A generalized minimal residual algorithm for solving nonsymmetric linear systems, SIAM J.Sci. Stat. Comput., 7 (1986), pp. 856-869, https://doi.org/10.1137/ 0907058.

[48] A. G. Salinger, N. M. Bou-Rabee, R. P. Pawlowsky, E. D. Wilkes, E. A. Burroughs, R. B. LehoucQ, AND L. A. Romero, LOCA 1.1. Library of Continuation Algorithms: Theory and Implementation Manual, Sandia National Laboratories, Albuquerque, NM, 2002.

[49] J. SÁnchez, F. Garcia, And M. NET, Critical torsional modes of convection in rotating fluid spheres at high Taylor numbers, J. Fluid Mech., 791 (2016), R1.

[50] J. SÁnchez, F. Garcia, AND M. NET, Radial collocation methods for the onset of convection in rotating spheres, J. Comput. Phys., 308 (2016), pp. 273-288.

[51] J. SÁnchez And M. NET, Numerical continuation methods for large-scale dissipative dynamical systems, Eur. Phys. J. Special Topics, 225 (2016), pp. 2465-2486.

[52] J. SÁnchez Umbría AND M. Net, Generation of bursting magnetic fields by nonperiodic torsional flows, Phys. Rev. E, 100 (2019), 053110.

[53] J. SÁnChez Umbría AND M. Net, Torsional solutions of convection in rotating fluid spheres, Phys. Rev. Fluids, 4 (2019), 013501. 
[54] P. Schmid And D. Henningson, Stability and Transition in Shear Flows, Appl. Math. Sci. 142, Springer, New York, 2000.

[55] R. Simitev And F. H. Busse, Prandtl-number dependence of convection-driven dynamos in rotating spherical fluid shells, J. Fluid Mech., 532 (2005), pp. 365-388.

[56] A. Tilgner And F. Busse, Finite amplitude convection in rotating spherical fluid shells, J. Fluid Mech., 332 (1997), pp. 359-376.

[57] H. Uecker, D. Wetzel, And J. RAdemacher, pde2path-a MATLAB package for continuation and bifurcation in $2 D$ elliptic systems, Numer. Math. Theory Methods Appl., 7 (2014), pp. 58-106.

[58] J. I. YANO, Asymptotic theory of thermal convection in a rapidly rotating system, J. Fluid Mech., 243 (1992), pp. 103-131.

[59] K. Zhang, Spiralling columnar convection in rapidly rotating spherical fluid shells, J. Fluid Mech., 236 (1992), pp. 535-556.

[60] K. Zhang, K. Lam, and D. Kong, Asymptotic theory for torsional convection in rotating fluid spheres, J. Fluid Mech., 813 (2017), R2. 